\title{
周期二阶线性微分方程复振荡 理论的一些结果*
}

\author{
高 任 安 \\ (华南师范大学数学系, 广州)
}

近年来, Bank, Laine, Gundersen, Langley 等人应用值分布论对二阶线性微分方程

$$
f^{\prime \prime}+A(z) f=0
$$

的复振荡理论做了许多研究工作, 并取得一系列有价值的结果 ${ }^{[1-6]}$. 本文进一步研究当 $A(z)$ 是整函数且是 $e^{\alpha x}$ ( $\alpha$ 是非零复常数) 的有理函数(即 $A(z)=B\left(e^{\alpha x}\right)-B(\zeta), B(\zeta)$ 是在 $0<$ $|\zeta|<\infty$ 内解析的有理函数)时方程 (1) 的复振荡问题及得到一些构成完整结论的结果. 易 知具整函数系数的方程 (1) 的解都是整函数. 下面以 $\lambda(f)$ 记函数 $f(z)$ 的零点序列的收玫指 数.

定理 1 设 $A(z)=B\left(e^{\alpha z}\right)$ 是周期为 $\omega=\frac{2 \pi i}{\alpha}$ 的周期整函数且是 $e^{\alpha z}$ 的有理函数, 则 以下性质成立:

(i) 若 $B(\zeta)$ 以 $\zeta=\infty$ 或 $\zeta=0$ 为奇数阶极点（包括那些经改变 $A(z)$ 的周期可变成这 种情况的), 如果方程 (1) 存在满足 $\lambda(f)<\infty$ 的非零解 $f(z)$, 则 $f(z)$ 与 $f(z+\omega)$ 线性无 关。

(ii) 若 $B(\zeta)$ 以 $\zeta=\infty$ 或 $\zeta=0$ 为偶数阶极点 (不包括那些经改变 $A(z)$ 的周期可变成奇 数阶极点情况的), 如果方程 (1) 存在满足 $\lambda(f)<\infty$ 的非零解 $f(z)$, 则 $f(z)$ 与 $f(z+\omega)$ 线 性相关.

证 若令 $a^{\prime}=-a$, 则 $B(\zeta)$ 的极点可化为 $B\left(t^{-1}\right)$ 的极点 $\infty$. 故若注意到 $f(x)=$ $k f(z-\omega)$ 与 $f(z+\omega)=k f(z)$ 等价, 易知只考虑 $\zeta=\infty$ 是 $B(\zeta)$ 的极点的情况已足够.

(i) 假定方程 (1) 存在满足 $\lambda(f)<\infty$ 的非零解 $f(z)$, 且 $f(z)$ 与 $f(z+\omega)$ 线性相关. 由文献 [3] 定理 $1, f(z)$ 可表为 $f(z)=e^{d z} G\left(e^{\alpha z}\right)$, 其中

$$
G(\zeta)=\psi(\zeta) \exp \left(\sum_{i=\mu}^{\nu} d_{i} \zeta^{i}\right),
$$

$\psi(\zeta)$ 是多项式, $\mu$ 及 $\nu$ 是整数 $(\mu \leqslant \nu), d, d_{i}$ 是常数且至少对某 $i \neq 0, d_{i} \neq 0$. 代入方程 (1) 得

$$
\alpha^{2} \zeta^{2} G^{\prime \prime}(\zeta)+\left(2 \alpha d+\alpha^{2}\right) \zeta G^{\prime}(\zeta)+\left[B(\zeta)+d^{2}\right] G(\zeta)=0 .
$$

因 $\zeta=\infty$ 是 $B(\zeta)$ 的奇数阶极点, 故 $B(\zeta)$ 可写为 $B(\zeta)=b_{p} \zeta^{p}+b_{p-1} \zeta^{p-1}+\cdots+b_{-k} \zeta^{-k}$, 其中 $P$ 是正奇整数, $k$ 是非负整数, $b_{p} \cdot b_{-k} \neq 0$. 由 (2) 式知 $\frac{G^{\prime}}{G}$ 及 $\frac{G^{\prime \prime}}{G}$ 是有理函数, 且易验 
当 $\zeta$ 接近 $\infty$ 时, 有

$$
\begin{aligned}
& \frac{G^{\prime}(\zeta)}{G(\zeta)}= \begin{cases}a \zeta^{-1}+O\left(|\zeta|^{-2}\right) & \text { 当 } \nu<1 \text { 时, } \\
b \zeta^{\nu-1}+O\left(|\zeta|^{p-2}\right) & \text { 当 } \nu \geqslant 1 \text { 时, }\end{cases} \\
& \frac{G^{\prime \prime}(\zeta)}{G(\zeta)}- \begin{cases}a(a-1) \zeta^{-2}+o\left(|\zeta|^{-3}\right) & \text { 当 } \nu<1 \text { 时, } \\
b^{2} \zeta^{2(\nu-1)}+O\left(|\zeta|^{20-3}\right) & \text { 当 } \nu \geqslant 1 \text { 时, }\end{cases}
\end{aligned}
$$

其中 $a, b$ 是非零常数. 将 (4) 及 (5) 式代人 (3) 式, 注意到 $2 v \neq p$, 易知当 $\zeta$ 足够大时, (3) 式不可能恒等地成立. 这就发生矛盾.

（ii）假定方程 (1) 存在满足 $\lambda(f)<\infty$ 的非零解 $f(z)$, 且 $f(x)$ 与 $f(z+\infty)$ 线性无关. 由文献 [3] 定理 1, $f(z)$ 可表为 $f(z)-e^{d z} G\left(e^{\frac{a}{2} z}\right)$, 其中

$$
G(\xi)=\phi(\xi) \exp \left(\sum_{i=\mu}^{\dot{d}} d_{i} \xi^{2 j+1}\right),
$$

$\boldsymbol{\psi}(\xi)$ 是多项式, $\mu$ 及 $\nu$ 是整数 $(\mu \leqslant \nu), d, d_{i}$ 是常数且至少有某 $d_{i} \neq 0$. 代人方程 (1) 得

$$
\text { - } \frac{a^{2}}{4} \xi^{2} G^{\prime \prime}(\xi)+\left(\alpha d+\frac{a^{2}}{4}\right) \xi G^{\prime}(\xi)+\left[B\left(\xi^{2}\right)+d^{2}\right] G(\xi)=0 .
$$

因 $\zeta=\infty$ 是 $B(\zeta)$ 的偶数阶极点, 故 $B\left(\xi^{2}\right)$ 可写为 $B\left(\xi^{2}\right)=b_{2 p} \xi^{4 p}+b_{2 p-1} \xi^{4 p-2}+\cdots+b_{-k} \xi^{-2 k}$, 其中 $p$ 是正整数, $k$ 是非负整数, $b_{2 p} \cdot b_{-k} \neq 0$. 由 (6) 式知 $\frac{G^{\prime}}{G}$ 及 $-\frac{G^{\prime \prime}}{G}$ 是有理函数, 且易验 证当 $\xi$ 接近 $\infty$ 时,有

$$
\begin{aligned}
& \frac{G^{\prime}(\xi)}{G(\xi)}= \begin{cases}a \xi^{-1}+O\left(|\xi|^{-2}\right) & \text { 当 } \nu<0 \text { 时, } \\
b \xi^{2 \nu}+O\left(|\xi|^{2 \nu-1}\right) & \text { 当 } \nu \geqslant 0 \text { 时, }\end{cases} \\
& \frac{G^{\prime \prime}(\xi)}{G(\xi)}- \begin{cases}a(a-1) \xi^{-2}+o\left(|\xi|^{-3}\right) & \text { 当 } \nu<0 \text { 时, } \\
b^{2} \xi^{4 \nu}+O\left(|\xi|^{4 \nu-1}\right) & \text { 当 } \nu \geqslant 0 \text { 时, }\end{cases}
\end{aligned}
$$

其中 $a, b$ 是非零常数。将 (8), (9) 式代人 (7) 式, 注意到 $2(2 v+1) \neq 4 p$, 易知当 $\xi$ 足解 大时 (7) 式不可能恒等地成立. 这就发生矛盾. 定理 1 证毕.

下面的定理 2 推广了文献 [3] 中的定理 3, 在文献 [3] 中要求 $\zeta=\infty$ 及 $\zeta=0$ 均是 $B(\zeta)$ 的奇数阶极点.

定玨 2 设 $A(z)=B\left(e^{\alpha x}\right)$ 是周期为 $\omega=\frac{2 \pi i}{\alpha}$ 的周期整函数且是 $e^{\alpha z}$ 的有理函数. 如 果 $\zeta=\infty$ 及 $\zeta=0$ 都是 $B(\zeta)$ 的极点, 且至少有一为奇数阶极点 (包括那些经改变 $A(z)$ 的周 期可变成这种情况的), 则方程 (1) 的任意非零解都有 $\lambda(f)-\infty$.

证 由文献 [3] 定理 3, 只需证明 $\zeta=\infty$ 及 $\zeta=0$ 中有一为 $B(\zeta)$ 的奇数阶极点, 另一 为 $B(\zeta)$ 的偶数阶极点的情况. 在此情况中, 假设方程 (1) 存在非零解 $f(z)$ 满足 $\lambda(f)<\infty$, 则由定理 $1, f(z)$ 与 $f(z+\infty)$ 既线性相关也线性无关. 这就发生矛盾. 定理 2 证毕.

下面例子说明定理 2 中“至少有一为奇数阶极点”的条件是必需的.

例 1. 函数 $f(x)=\exp \left(e^{2 x}+e^{-x}\right)$ 满足方程 $f^{\prime \prime}+\left(-4 e^{4 x}-4 e^{2 x}+4 e^{x}-e^{-x}-e^{-2 x}\right) f=$ 0. 显然 $\lambda(f)=0$.

下面的定理 3 大大推广了文献 [4] 中的定理 2. 在文献 [4] 中只指出当 $A(z)-e^{z}-K$ 时具有这个有䜵的性质.

定珪 3 设 $A(x)-B\left(e^{\alpha x}\right)$ 是 $e^{\alpha x}$ 的奇次多项式（包括那些经改变 $A(x)$ 的周期可变 
成这种情况的), 即 $B(\zeta)=\sum_{i=0}^{n} K_{i} \zeta^{i}$, 其中 $k$ 为正奇整数, $K_{k} \neq 0$. 若方程 (1) 存在满足 $\lambda(f)<\infty$ 的非零解 $f(z)$, 则必 $K_{0}=-\frac{\alpha^{2} q^{2}}{16}$, 其中 $q$ 为不小于 $k$ 的正奇整数. 反之, 当 $q$ 为形 式 $k(2 n+1)(n \geqslant 0)$ 的正奇整数时,方程 (1) 可能存在两个线性无关解 $f_{1} \neq \equiv 0, f_{2} \neq 0$ 满 是 $\lambda\left(f_{1}\right) \leqslant 1, \lambda\left(f_{2}\right) \leqslant 1$.

证 设方程 (1) 存在满足 $\lambda(f)<\infty$ 的非零解 $f(z)$, 则由定理 $1(i), f(x)$ 与 $f(z+\infty)$ 线性无关. 但由文献 [3] 引理 D, 函数 $E(z)=f(z) f(z+\omega)$ 满足 $E(z)^{2}-\phi\left(e^{\alpha x}\right)$, 其中 $\phi(\zeta)$ 是有理函数且在 $0<|\zeta|<\infty$ 内解析. 文献 [1] 3 证明 $E(z)$ 满足方程

$$
c^{2}-\left(E^{\prime}\right)^{2}+2 E E^{\prime \prime}+4 A E^{2}=0 \text {, }
$$

其中常数 $c \neq 0$ 是 $f(z)$ 与 $f(z+\infty)$ 的 Wronsky 行列式. 在 $(10)$ 式中以 $\phi(\zeta)$ 代替 $E(z)$ 得

$$
\alpha^{2} \zeta^{2} \phi \phi^{\prime \prime}-\frac{3}{4} \alpha^{2} \zeta^{2} \phi^{\prime 2}+\alpha^{2} \zeta \phi \phi^{\prime}+4\left(\sum_{i=0}^{k} K_{i} \zeta^{i}\right) \phi^{2}+c^{2} \phi=0 .
$$

由 (11) 式知当 $\zeta \rightarrow \infty$ 时, $4 \sum K_{i} \zeta^{i}+\frac{c^{2}}{\phi}$ 应趋于有限极限, 因此 $\phi(\zeta)$ 在 $\zeta=\infty$ 有一 $k$ 阶客 点. 于是, 注意到 $\phi(\zeta)$ 在 $\zeta=0$ 至多有一极点, 我们可将 $\phi(\zeta)$ 写为

$$
\phi(\zeta)=a_{k} \zeta^{-k}+\cdots+a_{q} \zeta^{-q},
$$

其中 $q \geqslant k, a_{k} \cdot a_{q} \neq 0$. 将 (12) 式代入 (11) 式并集中悬 $\zeta^{-2 q}$ 得

$$
\alpha^{2} q(q+1) a_{a}^{2}-\frac{3}{4} \alpha^{2} q^{2} a_{q}^{2}-\alpha^{2} q a_{q}^{2}+4 K_{0} a_{q}^{2}=0,
$$

由此得 $K_{0}=-\frac{\alpha^{2} q^{2}}{16}$. 其次, 我们有

$$
\begin{aligned}
E(z)^{2} e^{q \alpha z} & =a_{k} e^{(q-k) \alpha x}+\cdots+a_{q} \\
& =a_{k}\left(e^{\alpha z}-a_{1}\right) \cdots\left(e^{\alpha z}-a_{q-k}\right) .
\end{aligned}
$$

由于等式左边只可能有偶数阶零点, $\alpha_{i}$ 只可能偶数次出现, 故 $q-k$ 是偶数或零, 从而 $q$ 是 $\geqslant k$ 的正奇整数. 为了说明相反的情形, 只需看下例.

例 2. 设 $K_{0}=-\frac{a^{2} k^{2}(2 n+1)^{2}}{16}, n \geqslant 0$, 我们以 $a_{0}=1$,

$$
\left(4 m^{2} \alpha^{2} k^{2}+4 m \alpha^{2} k^{2}+16 K_{0}+\alpha^{2} k^{2}\right) a_{m}=16\left( \pm \sqrt{K_{k}} i\right)(m+1) \alpha k a_{m+1}
$$

来定义

$$
Q(\zeta)=\sum_{m=0}^{n} a_{m} \zeta^{m}
$$

容易验证函数 $w(z)-Q\left(e^{-\alpha k s / 2}\right)$ 满足方程

$$
w^{\prime \prime}+\left[2\left( \pm \sqrt{K_{k}} i\right) e^{\alpha k z / 2}-\frac{\alpha k}{2}\right] w^{\prime}+\left(\frac{\alpha^{2} k^{2}}{16}+K_{0}\right) w=0,
$$

因此, 函数

$$
f(z)=w(z) \exp \left[\frac{2}{\alpha k}\left( \pm \sqrt{K_{k} i}\right) e^{a k z / 2}-\frac{a k z}{4}\right]
$$


满足方程 $f^{\prime \prime}+\left(K_{k} e^{k a s}+K_{0}\right) f=0$. 易知 $\lambda(f) \leqslant 1$.

致晽：感谢中国科学院数学研究所何育兟教授对本文提供了宝贵意见.

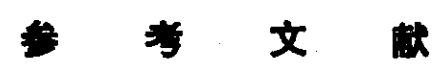

[1] Bank, S. and Laine, 1., Trans. Amer. Mazh. Soc., 273 (1982), 351-363.

[ 2 ]. Comment. Mazh. Hely, 58(1983), 656-677.

[3] - Representations of solutions of periodic second order linear differential equations (to be published).

[4] Bank, S., Laine, I. and Langley, J., On the frequency of zeros of solutions of second order linear differential equations (to appear in Result. Math).

[ 5] Gundersen, G., On the real zeros of solutions of $f^{\prime \prime}+\mathbf{A f}=0$, where $A$ is entire (to appear in Ann. Acad. Sci. Fenn.).

[6] Hellessten, S., Shen, L. and Williamson, J., Contcmporary Math, 25(1983), 63-67. 